\title{
GAMIFICANDO CON KAHOOT EN EVALUACIÓN FORMATIVA
}

\author{
Gamification with Kahoot en Formative Assessment \\ Gamificando com Kahoot na avaliaçao formativa
}

\begin{abstract}
Este estudio se ha llevado a cabo dentro del proyecto de I+D+i: "La competencias docentes en la formación inicial del profesorado de educación física”. Convocatoria de noviembre de 2013 del Programa Estatal de Investigación, Desarrollo e Innovación Orientada a los Retos de la Sociedad, en el marco del Plan Estatal de Investigación Científica y Técnica y de Innovación 2013-2016. Referencia: EDU 2013-42024-R. Duración: 3 años (2014-2016).
\end{abstract}

\section{Patricia Pintor Díaz}

Universidad de La Laguna, España. Teléfono: +34 922319643. Correo electrónico: ppindia@ull.edu.es

\section{Resumen}

Se trata de una experiencia basada en la gamificación de la evaluación formativa, mediante el uso de la aplicación digital Kahoot en la administración de feedback entre iguales. Fue aplicada en la asignatura "Educación y Desarrollo Psicomotor” del Grado de Maestro en Educación Infantil. Los resultados muestran gran satisfacción del alumnado por suponer un aprendizaje activo, que les permitió trabajar de forma colaborativa. También señalaron que el proceso de aprendizaje fue más motivador, se generaron aprendizajes significativos que ayudaron a relacionar la teoría con la práctica y aportaron retroalimentación.

Palabras clave: Evaluación Formativa; Gamificación; Kahoot

\begin{abstract}
Experience based on the gamification of the Formative Assessment, through the use of the Kahoot digital application in the administration of feedback between equals. It was applied in the subject "Education and Psychomotor Development" of the Degree in Infant Education. The results show great satisfaction of the students for assuming active learning, which allowed them to work collaboratively. They also pointed out that the
\end{abstract}


learning process was more motivating, generating significant learning that helped to relate the theory to practice and provided feedback.

Keywords: Formative Assessment; Gamification;Kahoot

\section{Resumo}

Experiência Gamificación com base na avaliação formativa, utilizando a aplicação digital de feedback gestão Kahoot pares. Foi aplicado em "Educação e Desenvolvimento Psicomotor" sujeito do Mestrado em Educação Infantil. Os resultados mostram alta satisfação dos estudantes, assumindo a aprendizagem ativo, permitindolhes trabalhar de forma colaborativa. Eles também observaram que o processo de aprendizagem foi mais motivador, aprendizagem significativa que ajudou a teoria ligação com a prática e forneceram feedback gerado.

Palavras-chave: Avaliaçao Formativa; Gamificacion; Kahoot

\section{Introducción}

La Gamificación consiste en trasladar la dinámica de los juegos al ámbito educativo (Gaitán, 2013). Esta goza de gran popularidad entre los entornos digitales, al aportar ingredientes muy atractivos para el alumnado (Moll, 2014); entre otros, se reconoce su capacidad para: motivar, fidelizar, fomentar trabajo colaborativo, etc. (Gaitán, 2013; Moll, 2014). Las dinámicas de gamificación más utilizadas son el uso de recompensas/regalos, fomento de la competición, consecución de estatus, cooperación, desafíos...

Reconocido su valor, no deja de ser una herramienta que debe ser convenientemente utilizada en el proceso educativo, para que este no pierda su esencia. Así pues, el reto que nos planteamos en esta experiencia es cómo vincularla a procesos de evaluación para que contribuya a su valor formativo. Con esta finalidad, integramos la gamificación en procesos de feedback entre iguales. Por un lado el feedback entre iguales permite a los estudiantes mantener un rol activo en su propio aprendizaje (Butler \& Winne, 1995) y por otro lado, la gamificación ayuda a que se sienta atraído y motivado; ambos factores favorecen la autorregulación del proceso de aprendizaje del alumnado. 


\section{Contextualización}

Se trata de una experiencia realizada en el curso 2016/17, con alumnado de la asignatura “Educación y Desarrollo Psicomotriz” de $3^{\circ}$ del Grado de Maestro en Educación Infantil de la Universidad de La Laguna. Se trata de una asignatura obligatoria, concentrada en 9 semanas, en la que se incide en la utilización de herramientas tecnológicas, y que ha sido aceptada dentro del "Programa de apoyo a la docencia presencial mediante herramientas TIC” de la mencionada universidad.

Como peculiaridad, señalaremos que esta asignatura tenía asignado en el horario del alumnado 3 h de clase /teórica el mismo día, lo que de antemano suponía un reto; para mantener la atención del alumnado, era preciso buscar recursos metodológicos atractivos, por lo que pensamos podía ser interesante la utilización de la aplicación Kahoot.

\section{Diseño y desarrollo}

El objetivo principal fue potenciar la motivación del alumnado mediante el uso de herramientas TIC en procesos de feedback entre iguales. Organizados en pequeños grupos (aproximadamente 5), debían elaborar un instrumento digital (presentación en Power Point, Prezi o similar) que sirviese como resumen de uno de los temas de la programación, de tal forma que, una vez concluido el tema, el grupo en cuestión era el encargado de exponer su trabajo y, tras el mismo, ayudar a sus compañeros/as a valorar sus conocimientos a través de un cuestionario Kahoot.

Optamos por utilizar un entorno digital que fuese más atractivo para el alumnado que el aula virtual Moodle, y diseñamos un Padlet, consistente en una pizarra o tablero que mostraba el contenido multimedia; con esta herramienta de la asignatura, aportamos el Feed-up de cada una de las tareas apoyándonos en recursos digitales que minimizaran la incertidumbre ante la utilización de aplicaciones que desconocían (Figura 1).

El cuestionario Kahoot debía ser revisado en tutoría antes de su exposición en clase. En lo que respecta a la calificación, en esta actividad era valorado el procedimiento que servía de resumen del tema y el cuestionario Kahoot para su evaluación.

Una vez finalizado el curso, el alumnado valoró la actividad a través del cuestionario de "Experiencia de buena práctica y su evaluación” diseñado por la Red 


\section{Interuniversitaria de Evaluación Formativa y Compartida en Docencia Universitaria, cuya tabla del apartado 4.1.1 reproducimos parcialmente (Tabla 1).}

\section{EVALUACIÓN KAHOOT}

\section{GUIA DEL TRABAJO GRUPAL}

- Diseñar cuestionario con 20 preguntas del tema asignado. Ver tutorial que se adjunta. Las preguntas serán incluidas en un banco de recursos para el examen final y se colgarán en este Padlet para consulta del alumnado.

- Enviarlo al profesor/a para su supervisión antes de exponerlo. - Implementarlo en el aula cuando finalice el tema.

- No se puede utilizar música, fotos o vídeos que no estén autorizados. La responsabilidad legal será de los autores/as del trabajo. Se adjuntan algunos enlaces para facilitar este trabajo.

- Finalmente, responder de forma grupal el cuestionario de

autoevaluación colgado en el aula virtual (Google form)
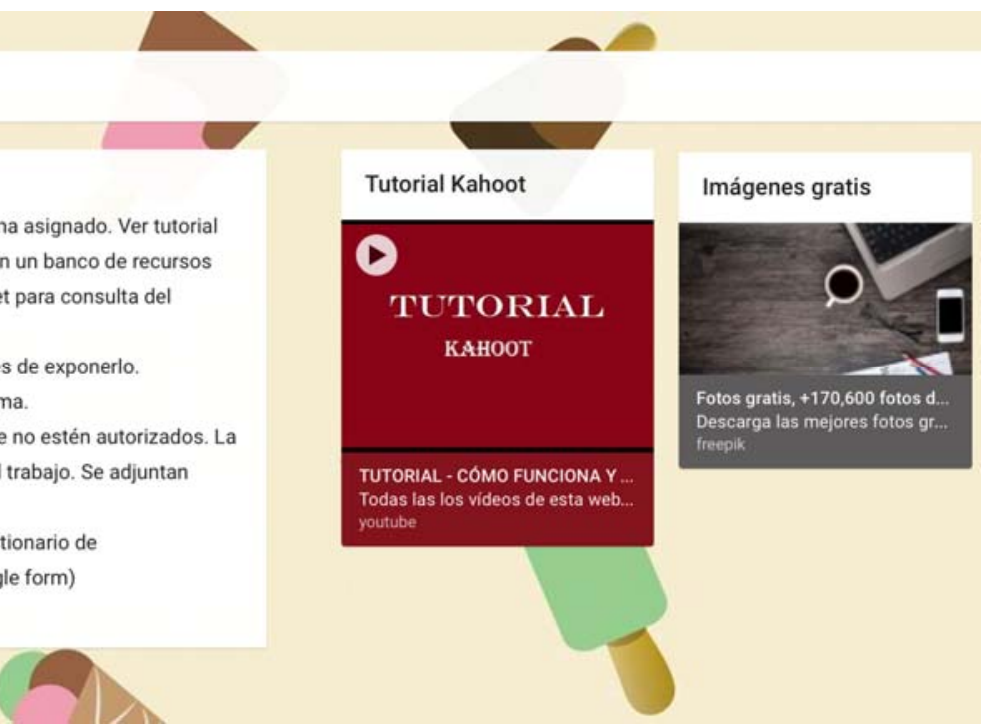

\section{Figura 1.}

Captura de pantalla de la sección del Padlet de la asignatura referente al Kahoot

\section{Tabla 1}

Cuestionario de "Experiencia de buena práctica y su evaluación” (parcial)

\begin{tabular}{|c|c|c|c|c|c|c|}
\hline \multirow[t]{2}{*}{ Items del cuestionario } & \multicolumn{5}{|c|}{$\begin{array}{l}\text { Poner una "X" en el nivel con un } \\
\text { porcentaje más alto en cada ítem. }\end{array}$} & \multirow[t]{2}{*}{$\begin{array}{c}\text { MEDIA } \\
(1-5)\end{array}$} \\
\hline & Nada & Poco & Algo & Bastante & Mucho & \\
\hline $\begin{array}{l}2-¿ \text { Crees que esta experiencia te ha ayudado a } \\
\text { adquirir competencias profesionales? }\end{array}$ & & & & $\mathrm{X}$ & $\mathrm{x}$ & 3,5 \\
\hline $\begin{array}{l}\text { 3-¿La evaluación que se ha planteado favorece } \\
\text { la adquisición de las competencias } \\
\text { profesionales }\end{array}$ & & & & $\mathrm{x}$ & & 3 \\
\hline $\begin{array}{l}\text { 4-¿Consideras útil lo aprendido con esta } \\
\text { experiencia? }\end{array}$ & & & & & $\mathrm{x}$ & 3.6 \\
\hline \multicolumn{7}{|l|}{$\begin{array}{l}\text { 5-¿¿Qué es lo más útil que has aprendido? Que } \\
\text { es una experiencia:.... }\end{array}$} \\
\hline $\begin{array}{l}\text { 5.1.-innovadora, porque desarrolla soluciones } \\
\text { nuevas o creativas }\end{array}$ & & & & $\mathrm{x}$ & & 3,1 \\
\hline $\begin{array}{l}\text { 5.2.-efectiva, porque demuestra un impacto } \\
\text { positivo y tangible de mejora }\end{array}$ & & & & & $\mathrm{x}$ & 3,3 \\
\hline $\begin{array}{l}\text { 5.3.-sostenible, porque se mantiene en el } \\
\text { tiempo y puede producir efectos duraderos }\end{array}$ & & & & $\mathrm{x}$ & $\mathrm{x}$ & 3,5 \\
\hline $\begin{array}{l}\text { 5.3.-replicable, cuando es posible utilizarla } \\
\text { como modelo para desarrollarla en otros } \\
\text { contextos }\end{array}$ & & & & & $\mathrm{x}$ & 3,5 \\
\hline $\begin{array}{l}\text { 8-Señala la satisfacción global en relación con } \\
\text { la experiencia }\end{array}$ & & & & $\mathrm{x}$ & $\mathrm{X}$ & 3,5 \\
\hline $\begin{array}{l}\text { 9-Señala la satisfacción global en relación con } \\
\text { la evaluación de la experiencia }\end{array}$ & & & & $\mathrm{x}$ & & 2,6 \\
\hline $\begin{array}{l}\text { 10-¿Cuál es el grado de dificultad de la } \\
\text { experiencia? }\end{array}$ & & & $\mathrm{X}$ & & & 2,3 \\
\hline
\end{tabular}

Gamificando con Kahoot en evaluación formativa 


\section{Evaluación y conclusiones}

El alumnado no estaba familiarizado con el uso del Kahoot, pero comentó resultarles muy sencillo al tratarse de una aplicación muy intuitiva; también agradeció contar con tutoriales de apoyo y guías en el Padlet de la asignatura.

Problemas encontrados: en la elaboración del artefacto, algunos grupos no destacaron lo sustantivo del tema. Planteamos como solución incidir en el feed-up, dejándolo claro en clase, elaborando un documento para el aula virtual y ampliando las sesiones de tutoría grupales. En lo que respecta al Kahoot, algunas preguntas fueron poco claras o las opciones de respuesta eran incorrectas. La solución a este problema no la vemos clara ya que tenemos poco tiempo para la revisión porque el tema se termina el viernes y el lunes en clase teórica se empieza con esta actividad. Asisten a tutoría durante esa semana pero el producto final lo vemos directamente en clase. Creemos que es importante que se haga antes de empezar el siguiente tema...así que como solución proponemos una revisión una vez expuesto en clase, antes de subirlas al aula virtual.

En lo que respecta a los resultados del cuestionario, el alumnado considera que la experiencia le supuso un aprendizaje activo, que les permitió trabajar de forma colaborativa, el proceso de aprendizaje fue más motivador, se generaron aprendizajes significativos, ayudando a relacionar la teoría con la práctica y existió retroalimentación.

Podemos concluir que la utilización de la aplicación Kahoot resultó positiva en el proceso de administración de feedback entre iguales, aportando un carácter motivador.

\section{Referencias}

Butler, D., \& Winne, P. (1995). Feedback and self-regulated learning: a theoretical synthesis. Review of Educational Research, 65(3), 245-281.

Espeso, P. (2016). Educación 3.0. Madrid: Revista Educación 3.0. Recuperado de http://www.educaciontrespuntocero.com/recursos/tutorial-crear-un-kahoot-paraclase/40146.html 
Espinosa, J. (2015). Educación 3.0. Madrid: Revista Educación 3.0. Recuperado de http://www.educaciontrespuntocero.com/experiencias/como-gamificar-el-aula-desecundaria/24888.html

Gaitán, V. (2013). E-ducativa. Alcalá de Henares: E-ducativa Educación Virtual S.A. Recuperado de http://www.educativa.com/blog-articulos/gamificacion-elaprendizaje-divertido/

Moll, S. (2014). Justifica tu respuesta. Recuperado de

http://justificaturespuesta.com/gamificacion-7-claves-para-entender-que-es-y-comofunciona/. 